\title{
An annotated checklist of the bryophytes of Taita Hills region, Kenya
}

\author{
Johannes Enroth, Tamás Pócs, Xiaolan He, Petri Nyqvist, \\ Åsa Stam, Itambo Malombe \& Jouko Rikkinen
}

An annotated checklist of the bryophytes of Taita Hills region, Kenya. - Acta Mus. Siles. Sci. Natur., 68: 53-66, 2019.

\begin{abstract}
Based on previous literature and our own collections, we list 285 bryophyte species (142 liverworts, 143 mosses) from the Taita Hills region (including Mt. Kasigau and Maktau Hill) in SE Kenya. New records for Kenya include the liverworts Archilejeunea elobulata Steph., Bazzania nitida (F. Weber) Grolle, Cololejeunea grossepapillosa (Horik.) N. Kitag., Diplasiolejeunea kraussiana (Lindenb.) Steph., D. villaumei Steph., Lejeunea amaniensis E.W. Jones, L. cyathearum E.W. Jones, Lopholejeunea laciniata E.W. Jones, Metzgeria crassipilis (Lindb.) A. Evans, M. nudifrons Steph., Plagiochila boryana (F. Weber) Nees, and P. moenkemeyeri Steph., and the mosses Leucophanes hildebrandtii Müll. Hal. and Neckeromnion lepineanum (Mont.) S. Olsson, Enroth, Huttunen \& D. Quandt. A further 22 liverworts and 13 mosses previously known from other parts of Kenya are reported for the first time from the Taita Hills region.
\end{abstract}

Key words: bryofloristics, Eastern Arc mountains, liverworts, mosses, sub-Saharan Africa

\section{Introduction}

Taita Hills in SE Kenya belong to a hotspot of global biodiversity (Myers et al. 2000). The bryophyte flora has been treated by Bytebier \& Chuah-Petiot (2002) and Enroth et al. (2013); those papers also include background information of the geology, vegetation and climate in the area. Malombe et al. (2016) focused on epiphyllous bryophytes on the mountains Chawia, Mbololo and Ngangao.

Bytebier \& Chuah-Petiot (2002) reported 85 moss species and 39 liverwort species from the Taita Hills. Enroth et al. (2013) added 43 moss species to the flora, raising the total number of bryophyte species known from the Taita Hills to 167. Twenty-nine liverworts and two mosses were subsequently added by Malombe et al. (2016). Based on our own recent collections and on previous literature, we report in this checklist 145 liverwort species and 143 moss species from the Taita Hills region (including the nearby Mt. Kasigau and Maktau Hill), so the total number of bryophyte species known from the region is 288 . Twelve of the liverworts and two of the mosses are first records for Kenya, and an additional 22 liverworts and 13 mosses are reported for the first time from the Taita Hills region. The liverworts in our own collections were identified by Tamás Pócs and Xiaolan He (fog net material, see below), and the mosses by Johannes Enroth. The distributions of African mosses by country were verified from the checklist by O'Shea (2006) and those of liverworts plus hornworts from the checklist by Wigginton (2018).

Most of the collections were made from the species' natural substrates (Figs. 1 and 2). However, in connection with the author Stam's PhD-project, we also identified the liverworts growing on plastic fog nets (raschel nets) placed in the forests in 2013 in order to collect data on the air moisture and its changes (Räsänen et al. 2018). The nets were in the forests for four years before they were collected. Mosses were not identified because they were poorly developed and, in stark contrast to liverworts, their numbers were generally negligible. The nets are deposited in $\mathrm{H}$ and their bryophyte and lichen cover and biomass will be described in more detail in a forthcoming paper (Stam et al., in prep.). 
In the checklist an asterisk (*) before a species name in boldface indicates a first record for Kenya. A species name in boldface but without an asterisk indicates a first published record for the Taita Hills, Mt. Kasigau or Maktau Hill. The specimens which the first records for Kenya and/or Taita Hills region are based on are cited in annotations numbered in superscript. Boldfaced names of the particular mountains in the Taita Hills denote first records for those mountains. Superscript ${ }^{a}$ means the species was reported from the mountain by Bytebier \& ChuahPetiot (2002), superscript ${ }^{b}$ means the species was previously reported from the mountain by Enroth et al. (2013), superscript ${ }^{\mathrm{c}}$ means the species was reported from the mountain by Malombe et al. (2016), and superscript ${ }^{\mathrm{d}}$ means the record is previously unpublished and now confirmed by T. Pócs. For the fog net material we use the following abbreviations: VUN = Vuria Upper North slope; VLS = Vuria Lower South slope; VMS = Vuria Middle South slope; VUOX = Vuria Open X; VUOY = Vuria Open Y; RSE = Research Station East; NGEW = Ngangao West side low E-slope; NGNE = Ngangao North E-side. The numbers after the abbreviations refer to the number of the net in the site. The abbreviation DP denotes "datapoint" and they are explained in the annotations. The abbreviation $\mathrm{JR}$ indicates the collector is Jouko Rikkinen. If a date is given with the JR-abbreviation, it means there are only photographs of the species from that location, but no voucher specimens.

\section{CHECKLIST}

\section{MARCHANTIOPHYTA}

(142 species)

Acanthocoleus chrysophyllus (Lehm.) Kruijt ${ }^{1}-$ Vuria

Acrolejeunea emergens (Mitt.) Steph. ${ }^{2}-$ Ngangao

Adelanthus lindenbergianus (Lehm.) Mitt. - Chawia ${ }^{\mathrm{c}}$

Aphanolejeunea microscopica (Taylor) A. Evans - Chawia ${ }^{\mathrm{c}}, \mathrm{Ngangao}^{\mathrm{c}}$

- var. exigua (A. Evans) A. Lücking \& Pócs - Ngangao $^{c}$

*Archilejeunea elobulata Steph. ${ }^{3}$ (Dibrachiella elobulata (Steph.) X.Q. Shi, R.L. Zhu \& Gradst.) - Mbololo

Bazzania decrescens (Lehm. \& Lindenb.) Trevis. - Mbololo ${ }^{\mathrm{a}}$

- subsp. pumila (Mitt.) Pócs - Mbololo ${ }^{\mathrm{a}}$

*Bazzania nitida (F. Weber) Grolle ${ }^{4}$ - Mbololo

Calypogeia fissa (L.) Raddi ${ }^{5}$ - Fururu, Mwachora, Vuria

Caudalejeunea hanningtonii (Mitt.) Schiffn. - Chawia ${ }^{\mathrm{c}}$, Mbololo ${ }^{\mathrm{c}}$, $\mathrm{Ngangao}^{\mathrm{a}}$

Caudalejeunea lehmanniana (Gottsche ex Gottsche, Lindenb. \& Nees) A. Evans - Chawia ${ }^{c}$, Mbololo ${ }^{\mathrm{c}}$, Ngangao $^{c}$

Cheilolejeunea cordistipula (Steph.) Grolle ex E.W. Jones - Mbololo a , Vuria (Stam VUS B2, VMN C3, VMS B2, VUOX C2)

Cheilolejeunea decursiva (Sande Lac.) R.M. Schust. ${ }^{6}$ - Vuria

Cheilolejeunea krakakammae (Lindenb.) R.M. Schust. - Chawia (Nyqvist 111764), Mbololo (Nyqvist 111357), Ngangao $^{c}$, Vuria $^{\text {a }}$

Cheilolejeunea montagnei (Gottsche) R.M. Schust. ${ }^{7}$ - Mbololo

Cheilolejeunea pocsii E.W. Jones - Mbololo ${ }^{\text {a }}$

Cheilolejeunea rigidula (Nees ex Mont.) R.M. Schust. - Fururu, Mbololo, Vuria

Cheilolejeunea rotundistipula (Lehm.) A. Evans ex Malombe $-\mathrm{Ngangao}^{\mathrm{c}}$

Cheilolejeunea trapezia (Nees) Mizut. - Chawia $^{\mathrm{c}}$, Mbololo ${ }^{\mathrm{c}}, \mathrm{Ngangao}^{\mathrm{c}}$

Cheilolejeunea unciloba (Lindenb.) Malombe - Mbololo ${ }^{\mathrm{a}}$

Cheilolejeunea usambarana (Steph.) Grolle - Mbololo a, Vuria (Stam VUN A4, VUN B3, VUN C3, VMN C2, VMN C3, VUOX C2, VUOX B3, VUOX C3, VUOY B3, VUOY C3)

Cheilolejeunea xanthocarpa (Lehm. \& Lindenb.) Malombe - Chawia ${ }^{\mathrm{c}}$, Fururu (Nyqvist 111353), Mbololo ${ }^{\mathrm{c}}$, Ngangao $^{\text {a, }}$ Vuria $^{\text {a }}$, Yale ${ }^{\text {a }}$ (as Leucolejeunea xanthocarpa (Lehm. \& Lindenb.) A. Evans)

Cololejeunea africana (Steph.) R.M. Schust. - Mbololo ${ }^{\text {a }}$

Cololejeunea appressa (A. Evans) Benedix - Chawia ${ }^{\mathrm{c}}$, Mbololo $^{\mathrm{c}}$

Cololejeunea bidentula (Steph.) E.W. Jones - Mbololo $^{\text {a }}$

Cololejeunea cardiocarpa (Mont.) A. Evans - Chawia ${ }^{\mathrm{c}}$, Mbololo ${ }^{\mathrm{a}}$, Ngangao ${ }^{\mathrm{c}}$, Vuria (Stam VUOX B3, VUOX C3), Wundanyi (Stam RSE B2, RSE C2, RSW B3).

Cololejeunea cristata (Steph.) R.M. Schust. - Chawia $^{\mathrm{c}}$, Mbololo $^{\mathrm{a}}, \mathrm{Ngangao}^{\mathrm{c}}$

Cololejeunea distalopapillata (E.W. Jones) R.M. Schust. - Chawia $^{c}$, Mbololo $^{a}$, Ngangao $^{c}$

Cololejeunea duvignaudii E.W. Jones - Mbololo ${ }^{\mathrm{a}}$

*Cololejeunea grossepapillosa (Horik.) N. Kitag. ${ }^{9}$ - Vuria

Cololejeunea hildebrandii (Austin) Steph. - Chawia ${ }^{\mathrm{c}}, \mathrm{Mbololo}^{\mathrm{a}}$, Ngangao ${ }^{\mathrm{a}}$

Cololejeunea jonesii Pócs - Ngangao ${ }^{\mathrm{c}}$

Cololejeunea lanceolata E.W. Jones - Chawia $^{\mathrm{c}}, \mathrm{Ngangao}^{\mathrm{c}}$

Cololejeunea lanciloba Steph. - Chawia ${ }^{\mathrm{c}}$, Mbololo ${ }^{\mathrm{a}}, \mathrm{Ngangao}^{\mathrm{c}}$ (all as C. tonkinensis) 

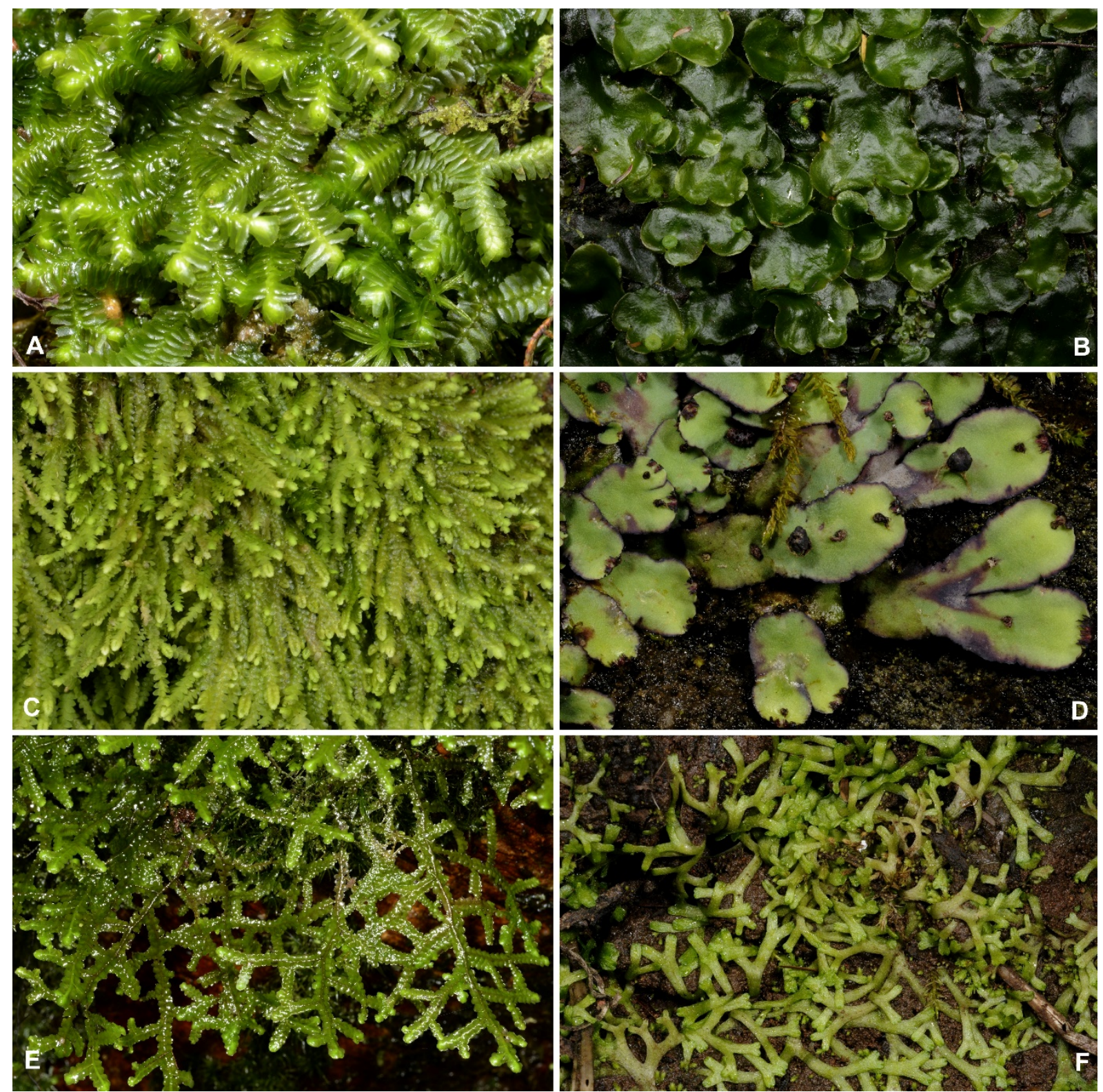

Fig 1: Liverworts photographed in situ in the Taita Hills. A - Bazzania descrecens ssp. pumila (JR13870). B - Dumortiera hirsuta (JR26580). C - Lejeunea flava (JR13848). D - Plagiochasma rupestre (JR51123). E - Radula boryana (JR13900). F - Riccia stricta (JR29604).

Cololejeunea latilobula (Herzog) Tixier - Chawia ${ }^{\mathrm{c}}$, Ngangao ${ }^{\mathrm{c}}$

Cololejeunea leloutrei (E.W. Jones) R.M. Schust. - Chawia $^{c}$, Mbololo $^{a}$, Ngangao $^{c}$

Cololejeunea malanjae Steph. - Chawia $^{\mathrm{c}}$, Mbololo $^{\mathrm{a}}$, Ngangao ${ }^{\mathrm{c}}$

Cololejeunea obtusifolia (E.W. Jones) Tixier - Chawia $^{\mathrm{c}}$, Mbololo $^{\mathrm{c}}$, Ngangao $^{\mathrm{c}}$

Cololejeuna occidentalis (E.W. Jones) Vanden Berghen - Mbololo ${ }^{c}$, Ngangao ${ }^{c}$

Cololejeunea pusilla Steph. - Chawia $^{\mathrm{c}}$, Mbololo $^{\mathrm{c}}$, Ngangao $^{\mathrm{c}}$

Cololejeunea tanzaniae Pócs - Chawia $^{c}$, Mbololo ${ }^{\mathrm{c}}$, Ngangao ${ }^{\mathrm{a}}$

Colura digitalis (Mitt.) Steph. - Chawia $^{c}$, Mbololo $^{a}$, Ngangao ${ }^{c}$

Colura dusenii (Steph.) Steph. - Mbololo $^{\mathrm{c}}$

Colura saroltae Pócs - Vuria (Pócs \& Váňa 2015).

Colura tenuicornis (A. Evans) Steph. - Chawia ${ }^{c}$, Mbololo ${ }^{c}$, Ngangao ${ }^{c}$, Vuria (Stam VUN B3, VLN B2, VLN B3, VLN C3, VLS D2, VMN B2, VMN C3, VUOX C2, VUOX B3, VUOX C3, VUOY B2, VUOY B3, VUOY C3)

Colura usambarica E.W. Jones - Mbololo a, Vuria (Stam VUN C3, VLN B2, VLN B3, VLN C3, VLS D2, VUOX C2, VUOX C3, VUOY C3) 
Diplasiolejeunea albifolia (Taylor) E.W. Jones - Chawia ${ }^{\mathrm{c}}, \mathrm{Mbololo}^{\mathrm{a}}$, Ngangao ${ }^{\mathrm{c}}$

Diplasiolejeunea cavifolia Steph. ${ }^{10}$ - Ngangao

Diplasiolejeunea cornuta Steph. - Chawia ${ }^{\mathrm{c}}, \mathrm{Mbololo}^{\mathrm{c}}, \mathrm{Ngangao}^{\mathrm{c}}$

*Diplasiolejeunea kraussiana (Lindenb.) Steph. ${ }^{11}$ - Ngangao, Wundanyi

Diplasiolejeunea runssorensis Steph. - Chawia ${ }^{\mathrm{c}}, \mathrm{Mbololo}^{\mathrm{a}}$, Ngangao ${ }^{\mathrm{c}}$

Diplasiolejeunea symoensii Vanden Berghen - Mbololo $^{\mathrm{d}}, \mathrm{Ngangao}^{\mathrm{d}}$

*Diplasiolejeunea villaumei Steph. - Ngangao ${ }^{\mathrm{d}}$

Diplasiolejeunea zakiae Tixier - Chawia ${ }^{\mathrm{c}}, \mathrm{Mbololo}^{\mathrm{a}}, \mathrm{Ngangao}^{\mathrm{a}}$

Drepanolejeunea cultrella (Mitt.) Steph. - Mbololo ${ }^{\mathrm{a}}$, Ngangao $^{\mathrm{c}}$

Drepanolejeunea madagascariensis (Steph.) Grolle - Mbololo ${ }^{\text {a }}$

Drepanolejeunea physaefolia (Gottsche) Steph. - Mbololo ${ }^{\text {a }}$, Ngangao ${ }^{\text {c }}$, Vuria (Stam VUN A4, VUN B3, VUN C3, VUS B2, VUS C2, VLN B2, VLN B3,VLN C3,VLS D2,VMN C2,VMN C3).

Drepanolejeunea pocsii Grolle - Chawia ${ }^{\mathrm{c}}, \mathrm{Mbololo}^{\mathrm{a}}$, Ngangao ${ }^{\mathrm{C}}$

Drepanolejeunea ternatensis (Gottsche) Steph. - Ngangao ${ }^{c}$

Drepanolejeunea trematodes (Nees) Bischl. - Mbololo ${ }^{\text {a }}$

Drepanolejeunea vesiculosa (Mitt.) Steph. ${ }^{12}-$ Mbololo

Dumortiera hirsuta (Sw.) Nees - Fururu (JR29191, 12. Jan. 2016), Kasigau (JR14359, 23. Oct. 2014; JR26580, 5. Oct. 2015; JR26587, 5. Oct. 2015), Mbololo a, Wundanyi ${ }^{\text {a, }}$, Vuria

Frullania angulata Mitt. - Chawia ${ }^{\mathrm{a}}$, Mbololo $^{\mathrm{a}}, \mathrm{Ngangao}^{\mathrm{a}}$, Vuria ${ }^{\mathrm{a}}$

Frullania apicalis Mitt. - Ngangao ${ }^{\mathrm{c}}$, Mbololo ${ }^{\mathrm{a}}$ - var. camerunensis Vanden Berghen - Mbololo ${ }^{\mathrm{a}}$

Frullania caffraria Steph. - Ngangao ${ }^{\text {a }}$

Frullania depressa Mitt. - Ngangao ${ }^{\mathrm{c}}$, Mbololo ${ }^{\mathrm{c}}$, Vuria (Stam VUN B3, VUN C3, VMN B2, VMN C2)

Frullania ericoides (Nees) Mont. - Chawia (Nyqvist 111336), Fururu (Nyqvist 111312), Ngangao (Stam NGNE A3), Vuria (Stam VUN A4, VUOX C2), Wundanyi ${ }^{a}$

Frullania obscura (Sw.) Dum. ${ }^{13}$ - Chawia, Ndiwenyi, Vuria (as F. arecae (Spreng.) Gottsche; Gradstein 2012)

Frullania obscurifolia Mitt. ${ }^{14}$ - Ngangao, Vuria, Wundanyi

Frullania serrata Gottsche - Vuria ${ }^{\text {a }}$

Frullania spongiosa Steph. ${ }^{15}$ - Ngangao

Frullania trinervis (Lehm.) Drege ${ }^{16}-$ Ngangao

Herbertus dicranus (Taylor ex Gottsche, Lindenb. \& Nees) Trevis. ${ }^{17}$ - Kasigau, Vuria

Heteroscyphus dubius (Gottsche) Schiffn. - Mbololo a ${ }^{\text {, Ngangao }}{ }^{\text {a }}$

Lejeunea acuta Mitt. - Ngangao ${ }^{\mathrm{c}}$, Mbololo ${ }^{\mathrm{a}}$

Lejeunea aloba Sande Lac. - Chawia $^{\mathrm{c}}, \mathrm{Mbololo}^{\mathrm{c}}, \mathrm{Ngangao}^{\mathrm{c}}$

*Lejeunea amaniensis E.W. Jones ${ }^{18}-$ Mbololo

Lejeunea anisophylla Mont. - Chawia $^{\mathrm{c}}$, Mbololo ${ }^{\mathrm{c}}$, Ngangao $^{\mathrm{c}}$, Ronge (Nyqvist 10460) (see Pócs 2010 for the taxonomy and nomenclature).

Lejeunea autoica R.M. Schust. - Chawia ${ }^{\mathrm{c}}$, Mbololo $^{\mathrm{c}}, \mathrm{Ngangao}^{\mathrm{c}}$

Lejeunea capensis Gottsche - Chawia ${ }^{a}$ (as L. caespitosa Lindenb.), Ngangao (Stam NGNE A3), Vuria (Stam VMS B2).

Lejeunea conformis (Mont. \& Nees) Malombe - Chawia (Nyqvist 111759), Vuria a

Lejeunea confusa E.W. Jones - Chawia ${ }^{\mathrm{c}}$, Mbololo ${ }^{\mathrm{C}}$, Ngangao ${ }^{\mathrm{c}}$, Vuria (Stam VUN A4, VUN C3, VMS B2)

*Lejeunea cyathearum E.W. Jones ${ }^{19}$ - Fururu, Ngangao

Lejeunea eckloniana Lindenb. - Chawia ${ }^{\mathrm{c}}$, Mbololo $^{\mathrm{c}}$, Ngangao $^{\mathrm{c}}$

Lejeunea flava (Sw.) Nees - Chawia ${ }^{\mathrm{c}}$, Mbololo ${ }^{\mathrm{c}}$, Ngangao ${ }^{\mathrm{a}}$, Vuria (Stam VUN A4, VUN B3, VUN C3, VUS B2, VUS C2, VLN B2, VLN B3, VLS D2, VMN C2, VMN C3, VMS B2, VUOX C2, VUOX B3), Wundanyi (Stam $R S W B 3, R S W C 3$, RSE B2, RSE C2)

Lejeunea grossecristata (Steph.) E.W. Jones - Chawia $^{c}$, Ngangao $^{c}$

Lejeunea hepaticola (Steph.) Steph. - Mbololo ${ }^{\mathrm{a}}, \mathrm{Ngangao}^{\mathrm{a}}, \mathrm{Vuria}^{\mathrm{a}}$

Lejeunea isophylla E.W. Jones - Mbololo (Nyqvist 10428), Vuria ${ }^{\text {a }}$

Lejeunea ramosissima Steph. - Mbololo ${ }^{\mathrm{c}}$

Lejeunea setacea (Steph.) Steph. - Chawia $^{c}$, Ngangao $^{\mathrm{c}}$

Lejeunea tabularis (Spreng.) Gottsche et al. - Chawia (Nyqvist 111764), Mbololo ${ }^{\mathrm{a}}$, Ngangao $^{\mathrm{c}}$, Vuria (Nyqvist 111812; Stam VUN B3, VUN C3, VUS B2, VUS C2, VMN C2, VMN C3, VUOY B3, VUOY C3)

Lejeunea villaumei (Steph.) Grolle ${ }^{20}$ - Fururu, Ngangao, Vuria.

Leptolejeunea maculata (Mitt.) Schiffn. - Mbololo ${ }^{a}$, Ngangao ${ }^{\mathrm{c}}$

Leptoschyphus expansus (Lehm.) Grolle - Mbololo ${ }^{\text {a }}$

Lophocolea bidentata (L.) Dumort. - Vuria ${ }^{\mathrm{a}}$

Lophocolea difformis Nees - Mbololo ${ }^{\text {a }}$

*Lopholejeunea laciniata E.W. Jones ${ }^{21}$ - Fururu 
Marchantia pappeana Lehm. - Fururu (JR29189, 12. Jan. 2016), Mwatate River a, Ngangao (JR29584, 16. Jan. 2016), Wundanyi (JR70981, 6. Sept. 2013; JR15391, 29. Oct. 2014; JR18651, 1. Feb. 2015; JR29208, 13. Jan. 2016)

Marchesinia excavata (Mitt.) Schiffn. - Vuria ${ }^{\text {a }}$

Marchesinia madagassa Steph. - Chawia (Nyqvist 111753), Mbololo a, Vuria (Stam VUN A4, VUN B3, VUN C3, VUS C3)

Metzgeria consanguinea Schiffn. - Chawia ${ }^{c}$, Mbololo $^{\mathrm{a}}$ (as M. attenuata Steph.; So 2004) Ngangao ${ }^{\mathrm{c}}$, Vuria (Stam VLN B2, VLN B3, VLN C3, VMN C2, VMN C3, VUOX B3, VUOX C3, VUOY B2, VUOY B3, VUOY C3)

*Metzgeria crassipilis (Lindb.) A. Evans ${ }^{22}-$ Mbololo

Metzgeria furcata (L.) Dumort. - Chawia ${ }^{c}$, Mbololo a (as M. decipiens \& M. australis; So 2004), Ngangao ${ }^{\text {c }}$, Vuria (Nyqvist 111824, 111825; Stam VUN A4, VLS D2), Wundanyi (Stam RSW B2, RSW B3, RSW C3, RSE B2, RSE C2

Metzgeria leptoneura Spruce - Ngangao ${ }^{c}$

Metzgeria madagassa Steph. - Mbololo (Nyqvist 10401), Vuria a (as M. limbato-setosa Steph.)

*Metzgeria nudifrons Steph. ${ }^{23}$ - Chawia, Mbololo

Metzgeria quadrifaria Steph. - Chawia ${ }^{\mathrm{c}}$

Microlejeunea africana Steph. - Chawia ${ }^{\mathrm{c}}$, Mbololo ${ }^{\mathrm{a}}$, Ndiwenyi (Nyqvist 111332), Ngangao ${ }^{\mathrm{c}}$, Vuria (Stam VUN $A 4, V U N C 3$, VUS C2, VLS D2, VMN C2, VMN C3, VMS B2), Wundanyi (Stam RSW B2, RSW B3, RSW C3, RSE C2)

Microlejeunea ankasica E.W. Jones - Mbololo $^{\mathrm{c}}, \mathrm{Ngangao}^{\mathrm{c}}$

Microlejeunea kamerunensis Steph. - Chawia ${ }^{c}$, Mbololo $^{\mathrm{c}}$, Ngangao $^{\mathrm{c}}$, Vuria (Stam VMN B2, VMS B3, VMS C3)

Myriocoleopsis minutissima (Sm.) R.L. Zhu, Y. Yu \& Pócs ${ }^{24}$ - Ngangao, Vuria, Wundanyi

Odontolejeunea lunulata (F. Weber) Schiffn. - Chawia ${ }^{\text {a }}$, Mbololo ${ }^{\text {a }}$, Ngangao ${ }^{\text {a }}$

Plagiochasma eximium (Schiffn.) Steph. - Ngangao ${ }^{\text {a }}$

Plagiochasma rupestre (J.R. Forst. \& G. Forst.) Steph. - Ngangao ${ }^{\text {a }}$

Plagiochila barteri Mitt. ${ }^{25}$ - Mbololo, Vuria

Plagiochila barteri Mitt. subsp. colorans (Steph.) Pócs - Vuria a

*Plagiochila boryana (F. Weber) $\mathrm{Nees}^{26}$ - Mbololo

Plagiochila effusa Steph. - Mbololo ${ }^{\text {a }}$, Vuria ${ }^{\text {a }}$

Plagiochila exigua (Taylor) Taylor ${ }^{27}$ - Vuria

Plagiochila fusifera Taylor - Mbololo a , Chawia (Nyqvist 111334), Vuria (Nyqvist 111806, 111809; Stam VUN A4)

Plagiochila integerrima Steph. - Mbololo ${ }^{\text {a }}$ Ngangao $^{\text {a }}$

Plagiochila kiaeri Gottsche ${ }^{28}-$ Mbololo

Plagiochila lastii Mitt. - Chawia ${ }^{\mathrm{a}}$, Mbololo ${ }^{\mathrm{a}}$, Ngangao ${ }^{\mathrm{a}}$

*Plagiochila moenkemeyeri Steph. ${ }^{29}$ - Chawia, Mbololo, Vuria

Plagiochila pectinata Willd. ex Lindenb. - Mbololo ${ }^{\text {a }}$

Plagiochila pseudoattenuata S.W. Arnell - Vuria ${ }^{a}$

Plagiochila punctata (Tayl.) Tayl. ${ }^{30}$ - Mbololo

Plagiochila squamulosa Mitt. var. sinuosa (Mitt.) Vanden Berghen - Chawia a , Fururu (Nyqvist 111324, 111750), Mbololo (Nyqvist 10428, 10453, 111376), Ndiwenyi (Nyqvist 111330, 111332), Ngangao ${ }^{\text {a }}$, Vuria ${ }^{\mathrm{a}}$ Plagiochila strictifolia Steph. - Mbololo ${ }^{\text {a }}$

Plagiochila terebrans Nees \& Mont. ex Lindenb. ${ }^{31}$ - Fururu, Ngangao, Vuria

Plicanthus hirtellus (F. Web.) R.M. Schust. ${ }^{32}$ - Vuria

Ptychanthus striatus (Lehm. \& Lindendb.) Nees - Mbololo ${ }^{\text {a }}$, Ngangao a (as P. africanus), Vuria (Nyqvist

111805). See Ahonen et al. (2005).

Radula ankefinensis Gottsche \& Steph. - Chawia $^{\mathrm{c}}$, Mbololo $^{\mathrm{a}}, \mathrm{Ngangao}^{\mathrm{a}}$

Radula boryana (F. Weber) Mont. - Mbololo ${ }^{\text {a }}$, Ngangao $^{\text {a }}$, Vuria ${ }^{\text {a }}$

Radula evelynae Yamada - Ngangao a, Vuria (Stam VMN C3, VUOY B3,

Radula flaccida Lindenb. \& Gottsche - Chawia ${ }^{\mathrm{c}}$, Mbololo ${ }^{\mathrm{c}}$, Ngangao (Stam NGNE A3, NGNE C2, NGNE C3, NGPW B3, NGPW C2, NGPW C3), Vuria (Stam VUN A4, VUN C3, VUS B2, VLS D2, VMS B2, VUOX C2)

Radula fulvifolia (Hook. f. \& Taylor) Gottsche et al. - Chawia ${ }^{\mathrm{a}}$, Macha ${ }^{\mathrm{a}}$, Mbololo ${ }^{\mathrm{a}}$, Mwachora ${ }^{\text {a }}$, Ngangao (Nyquist 111742)

Radula madagascariensis Gottsche - Mbololo ${ }^{\text {a }}$

Radula quadrata Gottsche - Chawia ${ }^{\mathrm{c}}$, Fururu (Nyqvist 111324), Mbololo a , Ngangao ${ }^{\text {a }}$, Vuria (Stam VLN B3, VLN C3), Yale ${ }^{\mathrm{a}}$

Radula stenocalyx Mont. - Mbololo a, Vuria (Stam VUN B3, VUS B2, VUS C2).

Radula voluta Taylor ex Gottsche et al. - Chawia a ${ }^{\text {a }}$ Mbololo a , Vuria (Nyqvist 111829; Stam VUN A4, VUN C3, VUS C2, VUS C3) 
Riccia stricta (Lindendb.) Perold - Ngangao ${ }^{\text {a }}$

Schiffneriolejeunea pappeana (Nees) Gradst. - Chawia (Nyqvist 111753), Mbololo ${ }^{\text {a }}$

\section{BRYOPHYTA}

(143 species)

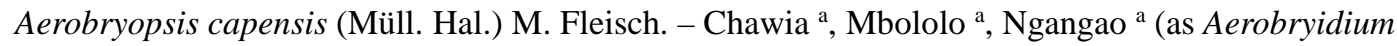
subpiligerum), Vuria (Nyqvist 111825)

Aerolindigia capillacea (Hornsch.) M. Menzel - Vuria ${ }^{\mathrm{b}}$

Alleniella complanata (Hedw.) S. Olsson, Enroth \& D. Quandt var. maxima (Dixon) - Mbololo ${ }^{\text {a }}$ (as Neckera c.)

Alleniella remota (Müll. Hal.) S. Olsson, Enroth \& D. Quandt - Chawia ${ }^{\mathrm{b}}$, Fururu ${ }^{\mathrm{b}}$, Mbololo ${ }^{\mathrm{b}}$, Ndiwenyi ${ }^{\mathrm{b}}$, Ngangao $^{b}$, Vuria $^{\mathrm{b}}$

Anomodon pseudotristis (Müll. Hal.) Kindb. - Kasigau ${ }^{\mathrm{b}}$

Atrichum androgynum (Müll. Hal.) A. Jaeger - Mbololo ${ }^{\text {a }}$

Barbella capillicaulis (Renauld \& Cardot) Cardot var. capillicaulis - Chawia ${ }^{\mathrm{b}}$, Ngangao ${ }^{\mathrm{b}}$, Vuria (Stam DP2)

Brachymenium nepalense Hook. ${ }^{33}$ - Chawia, Maktau Hill, Mwachora, Vuria

Braunia camptoclada P. de la Varde \& Thér. - Fururu ${ }^{\mathrm{b}}$

Breutelia diffracta Mitt. - Vuria ${ }^{\mathrm{a}}$

Breutelia stuhlmannii Broth. ${ }^{34}$ - Kasigau

Bryum argenteum Hedw. - Chawia (JR52643, 4. Dec. 2012), Fururu (Nyqvist 111305), Kishamba (JR26184, 3. Oct. 2015), Macha a ${ }^{\text {a }}$ Mbololo $^{\text {c }}$, Mwachora (JR71826, 9. Sept. 2013), Ngangao a, Vuria (JR51136, 14.

Sept. 2012), Wundanyi (JR32983, 30. Aug. 2011; JR53860, 9. Dec. 2012), Yale (JR71459, 8. Sept. 2013) Bryum bulbillicaule Müll. Hal. - "Taita Hills" a

Bryum huillense Welw. \& Duby - Mbololo ${ }^{a}$

Bryum keniae Müll. Hal. - Mbololo ${ }^{\text {a }}$, Ngangao ${ }^{\text {a }}$, Vuria ${ }^{\text {a }}$, Yale ${ }^{\text {a }}$

Bryum preussii Broth. - Vuria ${ }^{\text {a }}$

Bryum pseudotriquetrum (Hedw.) P. Gaertn. , B. Mey. \& Scherb. var. pseudotriquetrum - Macha ${ }^{\text {a }}$, Mwachora ${ }^{\mathrm{a}}$, Ngangao ${ }^{\text {a }}$, Yale ${ }^{\text {a }}$

Bryum taitae Müll. Hal. - "Taita Hills" a

Calymperes tenerum Müll. Hal. - Chawia ${ }^{\mathrm{c}}$, Mbololo ${ }^{\mathrm{c}}$

Campylopus arctocarpus (Hornsch.) Mitt. subsp. madecassus (Besch.) J.-P. Frahm - Mbololo (Nyqvist 10434), Vuria $^{\mathrm{b}}$

Campylopus crateris Besch. - Mbololo ${ }^{\mathrm{b}}$

Campylopus dicranoides Thér. - Fururu (Nyqvist 111333), Mbololo a ${ }^{\text {, Ngangao }}{ }^{\text {a }}$

Campylopus flexuosus (Hedw.) Brid. var. incacorralis (Herzog) J.-P. Frahm - Fururu (Nyqvist 111793), Mbololo (Nyqvist 10402, 10405), Ngangao ${ }^{\text {a }}$

Campylopus hildebrandtii (Müll. Hal.) A. Jaeger - Kasigau (JR14338), Vuria ${ }^{\mathrm{b}}$

Campylopus johannis-meyeri (Müll. Hal.) Kindb. - Fururu ${ }^{\mathrm{b}}$

Campylopus nanophyllus Müll. Hal. ex Broth. - Ngangao ${ }^{\text {b }}$, Yale (Stam DP175)

Campylopus nivalis (Brid.) Brid. var. nivalis - Vuria ${ }^{\text {a }}$

Campylopus perichaetialis P. de la Varde \& Thér. - Fururu (Nyqvist 111792), Mbololo a

Campylopus pilifer Brid. subsp. pilifer - Fururu (Nyqvist 111310, 111315, 111333), Vuria a

Campylopus savannarum (Müll. Hal.) Mitten - Ngangao ${ }^{a}$

Campylopus thwaitesii (Mitt.) A. Jaeger - Fururu b, Kasigau (JR10K54A, JR10K584B)

Chenia leptophylla (Müll. Hal.) R.H. Zander ${ }^{35}-$ Kasigau

Cryphaea rutenbergii Müll. Hal. ${ }^{36}$ - Maktau Hill, Vuria, Yale (Stam DP71, DP73)

Cyclodictyon borbonicum (Besch.) Broth. - Mbololo ${ }^{\text {a }}$, Yale ${ }^{\text {a }}$

Cyclodictyon dixonianum Demaret - Mbololo $^{\text {a }}$

Cyclodictyon krebedjense Broth. var. argutidens P. de la Varde - Fururu (Nyqvist 111791), Chawia ${ }^{\text {a }}$

Cyclodictyon vallis-gratiae (Müll. Hal.) Kuntze - Mbololo a, Vuria (Nyqvist 111816)

Dicranoloma billardieri (Brid.) Paris - Kasigau (JR10K54B), Vuria ${ }^{\text {a }}$

Dicranum johnstonii Mitt. - Vuria ${ }^{\text {a }}$

Ditrichum difficile (Duby) M. Fleisch. - Mbololo ${ }^{\mathrm{a}}$

Ectropothecium regulare (Brid.) A. Jaeger - Ngangao ${ }^{b}$

Entodontopsis nitens (Mitt.) W.R. Buck \& Ireland - Chawia ${ }^{b}$

Entosthodon hildebrandtii Müll. Hal. - "Taita Hills" a

Erpodium beccarii Müll. Hal. ex Venturi - Kasigau ${ }^{\mathrm{b}}$ 

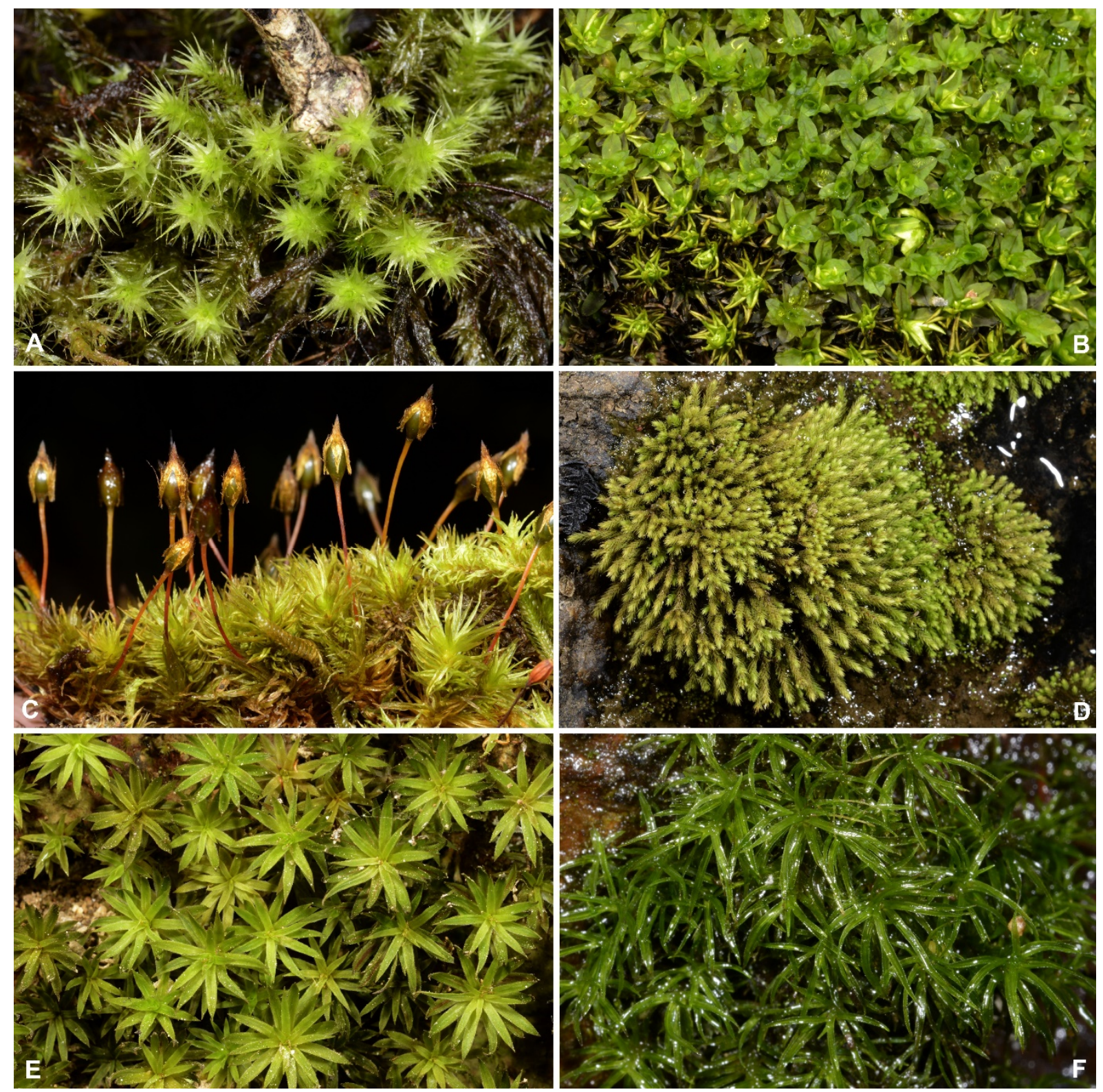

Fig 2: Mosses photographed in situ in the Taita Hills. A - Breutelia stuhlmannii (JR13902). B - Hyophila involuta (JR14198). C - Macromitrium sulcatum (JR14309). D - Philonotis hastata (JR18624). E - Pogonatum belangeri (JR13419). F - Syrrhopodon asper (JR13905).

Erythrodontium subjulaceum (Müll. Hal.) Paris - Vuria ${ }^{\text {a }}$

Fissidens androgynus Bruch ${ }^{37}$ - Kasigau

Fissidens asplenioides Hedw. - Mbololo ${ }^{\mathrm{a}}$, Vuria (JR71821)

Fissidens bogosicus Müll. Hal. - Ngangao ${ }^{\mathrm{a}}$

Fissidens borgenii Hampe - Kasigau ${ }^{\mathrm{b}}$

Fissidens intramarginatus (Hampe) Mitten - Fururu (Nyqvist 111327), Chawia ${ }^{\mathrm{a}}$, Macha $^{\mathrm{a}}$, Mbololo $^{\mathrm{a}}$, Ngangao $^{\mathrm{a}}$ Fissidens leucocinctus Hampe - Mbololo ${ }^{\mathrm{b}}$, Vuria ${ }^{\mathrm{b}}$

Fissidens megalotus Schimp. ex Müll. Hal. ssp. helictocaulos (Müll. Hal.) Brugg.-Nann. - "Taita Hills", (Kis 1985)

Fissidens ovatus Brid. ${ }^{38}$ - Vuria

Fissidens sciophyllus Mitt. - Ngangao ${ }^{\mathrm{a}}$

Fissidens splendens Brugg.-Nann. - Mbololo ${ }^{\mathrm{b}}$

Fissidens submarginatus Bruch ex C. Krauss - Mbololo ${ }^{a}$

Fissidens usambaricus Broth. - Macha ${ }^{\mathrm{a}}$

Fissidens weirii Mitt. - Macha ${ }^{\mathrm{a}}$, Mbololo ${ }^{\mathrm{a}}$ 
Floribundaria floribunda (Dozy \& Molk.) M. Fleisch. - Chawia ${ }^{\text {a }}$, Mbololo ${ }^{\text {a }}$

Floribundaria vaginans (Welw. \& Duby) Broth. - Chawia ${ }^{\mathrm{b}}$, Mbololo $^{\mathrm{b}}$, Ngangao (Stam DP142)

Forsstroemia producta (Hornsch.) Paris - Kasigau (JR10K095B), Mbololo a, Ngangao a, Vuria (Stam DP42), Wundanyi ${ }^{\text {a }}$, Yale (Stam DP78, DP86)

Funaria hygrometrica Hedw. ${ }^{39}$ - Yale

Hildebrandtiella phleoides (Desv. ex Brid) B.H. Allen \& Magill - Chawia ${ }^{\text {b }}$, Kasigau (JR10073, JR13824), Mbololo $^{\mathrm{b}}$, Ngangao $^{\mathrm{b}}$

Homaliodendron piniforme (Brid.) Enroth - Chawia $^{\mathrm{b}}$, Mbololo $^{\mathrm{b}}$, Kasigau ${ }^{\mathrm{b}}$

Hookeriopsis ambigua P. de la Varde - Chawia ${ }^{\mathrm{b}}$, Kasigau ${ }^{\mathrm{b}}$

Hyophila involuta (Hook.) A. Jaeger ${ }^{40}$ - Kasigau (JR10K353A)

Hypnum cupressiforme Hedw. - Chawia ${ }^{\text {a }}$, Ngangao (Nyqvist 111739), Vuria ${ }^{\text {a }}$

Hypopterygium tamarisci (Sw.) Brid. ex Müll. Hal. - Chawia a Kasigau (JR13790, JR10K139A), Mbololo a, Ngangao $^{\text {a }}$ (as H. mildbraedii Broth.), Vuria (JR50964, 14. Sept. 2012)

Jaegerina stolonifera (Müll. Hal.) Müll. Hal. subsp. stolonifera - Ngangao a

Lepidopilum lastii Mitt. - Ngangao (Stam DP104), Vuria ${ }^{\mathrm{b}}$

Leptodon smithii (Hedw.) F. Weber \& D. Mohr - Vuria (Stam DP48), Wundanyi a , Yale (Stam DP86)

Leptodontium longicaule Mitt. var. microuncinatum (Dusén) R.H. Zander - Wundanyi a

Leptodontium viticulosoides (P. Beauv.) Wijk \& Margad. var. viticulosoides - Ngangao ${ }^{\text {a }}$, Vuria ${ }^{\text {a }}$, Wundanyi ${ }^{\text {a }}$, Yale $^{\mathrm{a}}$

Leucobryum isleanum Besch. var. molle (Müll. Hal.) Cardot - Kasigau (JR13820, JR19K296, JR10K512), Mbololo $^{\text {a }}$, Ngangao ${ }^{\text {a }}$

Leucodon sciuroides (Hedw.) Schwägr.) var. sciuroides - Vuria ${ }^{\mathrm{b}}$

Leucoloma aspericuspis $\mathrm{P}$. de la Varde - Mbololo ${ }^{\text {a }}$

Leucoloma rutenbergii (Geh.) C.H. Wright var. elatum Renauld - Mbololo ${ }^{\mathrm{b}}$

Leucoloma zuluense Broth. \& Bryhn var. zuluense - Chawia ${ }^{\mathrm{b}}$, Mbololo ${ }^{\mathrm{b}}$, Ngangao $^{\mathrm{b}}$

*Leucophanes hildebrandtii Müll. Hal. ${ }^{41}$ - Kasigau.

Lindbergia patentifolia Dixon - Chawia ${ }^{\text {a }}$

Lopidium struthiopteris (Brid.) M. Fleisch. - Kasigau (JR10K156A), Mbololo a (as L. penniforme (Thunb. ex Brid.) M. Fleisch.; cf. Kruijer 2002), Ngangao (Nyqvist 10482)

Macrocoma abyssinica (Müll. Hal.) Vitt - Mbololo ${ }^{\text {a }}$

Macrocoma tenuis (Hook. \& Grev.) Vitt - Fururu (Nyqvist 111792), Kasigau (JR13759, JR10K064, JR10K095, JR10K383), Maktau Hill (JR14584, 14595), Mbololo a , Ngangao (Nyqvist 10467, 10478, 112202, 111715),

Vuria (Nyqvist 111802)

Macrohymenium acidodon (Mont.) Dozy \& Molk. - Vuria ${ }^{\text {a }}$

Macromitrium rufescens Besch. - Vuria ${ }^{\mathrm{a}}$

Macromitrium sulcatum (Hook.) Brid. - Kasigau (JR14309), Mbololo ${ }^{\text {a }}$, Ngangao ${ }^{\text {a }}$

Mittenothamnium reptans (Hedw.) Cardot - Chawia (Nyqvist 111754), Fururu (Nyqvist 111320, 111321), Mbololo (Nyqvist 10447), Ngangao (Nyqvist 111722), Vuria ${ }^{\text {a }}$

Neckera platyantha (Müll. Hal.) Paris - Mbololo ${ }^{\mathrm{a}}$, Ngangao ${ }^{\mathrm{a}}$, Yale ${ }^{\mathrm{a}}$

*Neckeromnion lepineanum (Mont.) S. Olsson, Enroth, Huttunen \& D. Quandt ${ }^{42}$ - Kasigau

Neorutenbergia usagarae (Dixon) Bizot \& Pócs - Mbololo a , Kasigau (JR10K559C, JR10K584), Ngangao (Stam DP107, DP108)

Nogopterium gracile (Hedw.) Crosby \& W.R. Buck - Ngangao a, Yale (JR15255, JR15298).

Orthorrhynchidium planifrons (Renauld \& Paris) Renauld \& Cardot - Mbololo ${ }^{\mathrm{b}}$ Ngangao $^{\mathrm{b}}$ (as Calyptothecium planifrons)

Orthostichella capillicaulis (Müll. Hal. ex Kindb.) B.H. Allen \& Magill - Fururu ${ }^{\text {b }}$, Kasigau ${ }^{\text {b }}$, Mbololo ${ }^{\text {, }}$, Ngangao $^{\mathrm{b}}$, Vuria $^{\mathrm{b}}$

Orthostichella pandurifolia (Müll. Hal.) W.R. Buck ${ }^{43}$ - Chawia ${ }^{\text {a }}$, Kasigau (JR1OK254B), Kichuchenyi ${ }^{\text {a }}$, Macha ${ }^{\text {a }}$, Mbololo ${ }^{\text {a }}$, Mwachora ${ }^{\text {a }}$, Ngangao ${ }^{\text {a, }}$, Vuria ${ }^{\text {a }}$, Yale ${ }^{\text {a }}$

Orthostichella rigida (Müll. Hal.) B.H. Allen \& Magill - Chawia ${ }^{c}$, Fururu (Nyqvist 111313, 111797),

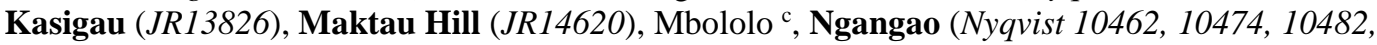
111714), Vuria (Nyqvist 111802, 111805, 111807, 111817, 111819), Yale (Stam DP70, DP71)

Orthostichopsis subimbricata (Hampe) Broth. ${ }^{44}$ - Kasigau, Vuria

Palamocladium leskeoides (Hook.) E. Britton - Mbololo (Nyqvist 10444), Vuria (Nyqvist 111802, 111824), Yale $^{\text {a }}$

Papillaria africana (Müll. Hal.) A. Jaeger - Chawia a , Fururu (Nyqvist 111324, 111787), Kasigau (JR10K245A), Mbololo (Nyqvist 10462), Ngangao a, Vuria (Stam DP48), Yale ${ }^{\text {a }}$

Pelekium varians (Welw. \& Duby) Touw - Kasigau ${ }^{\mathrm{b}}$

Pelekium versicolor (Hornsch. ex Müll. Hal.) A. Touw - Ngangao a (as Thuidium versicolor; cf. Touw 2001)

Philonotis curvula (Müll. Hal.) Kindb. - "Taita Hills" a 
Philonotis fontana (Hedw.) Brid. - Yale ${ }^{\text {a }}$

Philonotis hastata (Duby) Wijk \& Margad. ${ }^{45}$ - Wundanyi

Pilopogon africanus Broth. - Mbololo ${ }^{\text {a }}$

Pilotrichella ampullacea (Müll. Hal.) A. Jaeger - Mbololo a ${ }^{\text {, Vuria }}{ }^{\text {a }}$, Yale ${ }^{\text {a }}$

Plagiothecium nitens Dixon - Vuria ${ }^{\text {a }}$

Pogonatum belangeri (Müll. Hal.) A. Jaeger - Mbololo ${ }^{\text {b }}$, Ngerenyi (between Wundanyi and Chawia; JR13419).

Pogonatum urnigerum (Hedw.) P. Beauv. ${ }^{46}-$ Mbololo $^{\text {a }}$

Polytrichum commune Hedw. - Ngangao a, Vuria ${ }^{\text {a }}$

Polytrichum subpilosum P. Beauv. - Vuria ${ }^{\mathrm{a}}$

Porotrichodendron madagassum (Kiaer ex Besch.) S. Olsson, Enroth \& D. Quandt - Vuria ${ }^{\mathrm{b}}$

Porotrichum elongatum (Welw. \& Duby) A. Gepp - Chawia ${ }^{\mathrm{b}}$, Mbololo $^{\mathrm{b}}$, Ngangao $^{\mathrm{b}}$, Vuria ${ }^{\mathrm{b}}, \mathrm{Kasigau}^{\mathrm{b}}$

Porotrichum stipitatum (Mitt.) W.R. Buck - Chawia (Nyqvist 111350), Fururu (Nyqvist 111315), Kasigau $(J R 10 K 156 B)$, Mbololo $^{\text {a }}$, Ngangao $^{\text {a }}$, Yale $^{\text {a }}$

Porotrichum usagarum Mitt. - Chawia (Nyqvist 111343, 111345), Mbololo a, ${ }^{\text {Ngangao }}{ }^{\text {a }}$, Vuria ${ }^{\text {a }}$

Prionodon ciliatus Besch. - Vuria ${ }^{\text {a }}$

Pterobryon flagelliferum Mitt. - Chawia (Nyqvist 111350), Kasigau (JR14348, JR10K139B, JR10K142A, JR10K302A; JR13910, 22. Oct. 2014; JR13926, 22. Oct. 2014), Mbololo a, Ngangao a

Pterobryopsis hoehnelii (Müll. Hal.) Magill - Mbololo (Nyqvist 111387), Ngangao a (as Calyptothecium hoehnelii (Müll. Hal.) Argent), Vuria (Nyqvist 111815)

Pyrrhobryum spiniforme (Hedw.) Mitt. - Chawia a , Kasigau (JR10K273B, JR10K504B, JR10K52A, JR10K533B, JR10K573A), Mbololo a , Ngangao ${ }^{\text {a }}$

Racopilum africanum Mitt. - Chawia ${ }^{\text {a }}$ Kasigau (JR13850, JR10K498B), Kichuchenyi ${ }^{\text {a }}$, Macha ${ }^{\text {a }}$, Mbololo ${ }^{\text {a, }}$ Mwachora ${ }^{\text {a }}$ Ngangao $^{\text {a }}$, Yale ${ }^{\text {a }}$

Renauldia lycopodioides Bizot ex Pócs - Chawia ${ }^{\mathrm{a}}$, Macha ${ }^{\mathrm{a}}$, Mbololo $^{\mathrm{a}}$, Mwachora ${ }^{\mathrm{a}}, \mathrm{Ngangao}^{\mathrm{a}}$

Rhacopilopsis trinitensis (Müll. Hal.) E. Briton ex Dixon - Mbololo ${ }^{\mathrm{b}}$ Ngangao $^{\mathrm{b}}$

Rhacopilopsis variegata (Welw. \& Duby) M.C. Watling \& O'Shea - Kasigau (JR1OK32A), Mbololo ${ }^{\mathrm{b}}$, Ngangao $^{\text {b }}$

Rhizofabronia perpilosa (Broth.) Broth. - Yale $^{\mathrm{a}}$

Rhizofabronia persoonii (Schwägr.) M. Fleisch. var. sphaerocarpa (Dusén) Bizot ex Ochyra - Vuria ${ }^{\text {a }}$

Rhodobryum ontariense Kindb. ${ }^{47}$ - Kasigau, Ngangao, Vuria, Yale

Rhynchostegiella holstii (Broth.) Broth. ${ }^{48}$ - Ngangao

*Rhynchostegium buluense (Broth.) Paris ${ }^{49}-$ Vuria

Rhynchostegium comorae (Müll. Hal.) A. Jaeger - Fururu ${ }^{\mathrm{b}}$, Ngangao $^{\mathrm{b}}$, Vuria (Stam DP40), Wundanyi ${ }^{\mathrm{b}}$, Yale (Stam DP84)

Rigodium toxarion (Schwägr.) A. Jaeger var. toxarion ${ }^{50}-$ Vuria

Schimperella bello-intricata (Müll. Hal. ex Broth.) W.R. Buck - Chawia ${ }^{\text {b }}$

Schlotheimia percuspidata Müll. Hal. - Fururu ${ }^{\mathrm{b}}$, Mbololo ${ }^{\mathrm{b}}$

Schoenobryum robustum (Broth.) Manuel - Chawia (Nyqvist 111342, 111347, 111761), Mbololo a , Ngangao

(Nyqvist 10467), Vuria Stam DP16, JR10082), Yale (Stam DP77, DP97)

Sematophyllum brachycarpum (Hampe) Broth. - Mbololo ${ }^{\text {a }}$, Ngangao ${ }^{\text {a }}$

Sematophyllum elgonense (Dixon) Broth. - Fururu ${ }^{\mathrm{b}}$, Ronge $^{\mathrm{b}}$

Sematophyllum subpinnatum (Brid.) E. Britton - Mbololo ${ }^{\mathrm{a}}$, Ngangao ${ }^{\mathrm{a}}$

Squamidium brasiliense (Hornsch.) Broth. - Chawia a , Fururu (Nyqvist 111303, 111752), Mbololo ${ }^{\text {a }}$, Ngangao (Nyqvist 10467), Vuria ${ }^{\mathrm{a}}$, Yale ${ }^{\mathrm{a}}$

Syntrichia fragilis (Taylor) Ochyra - "Taita Hills" a, Vuria (Stam DP48)

Syrrhopodon asper Mitt. - Kasigau (JR13905), Mbololo ${ }^{\text {a, b}, ~ N g a n g a o ~}{ }^{\text {b }}$ (Bytebier \& Chuah-Petiot as $S$. usambaricus)

Syrrhopodon gaudichaudii Mont. - Fururu ${ }^{\mathrm{b}}$, Vuria $^{\mathrm{b}}$

Trachyphyllum inflexum (Harv.) A. Gepp - Kasigau ${ }^{\mathrm{b}}$

Trachypus bicolor Reinw. \& Hornsch. var. viridulus (Mitt.) Zanten - Mbololo ${ }^{\mathrm{b}}$

Trichostomum brachydontium Bruch - Kasigau (JR10K463A), Ngangao a

Wijkia trichocolea (Müll. Hal.) H.A. Crum - Kasigau (JR10K32B, JR10K44A, JR10K512B, JR10K533C, JR10K567), Mbololo ${ }^{\text {a }}$, Ngangao ${ }^{\text {a }}$

Acknowledgements: We are grateful for the opportunity to dedicate this paper to our bryologist colleagues and friends Dr. Halina Bednarek-Ochyra and Dr. Ryszard Ochyra on the occasion of their $60^{\text {th }}$ and $70^{\text {th }}$ birthdays, respectively. We thank Kenya Forestry Service for access permits and direction in the forests. 


\section{References}

Ahonen I., Sass-Gyarmati A. \& Pócs T. (2005): Molecular, morphological and taxonomic evaluation of the Ptychanthus striatus (Lejeuneaceae, Marchantiophyta) complex. - Acta Botanica Hungarica 47: 226-246.

Allen B. \& Magill R.E. (2007): A revision of Orthostichella (Neckeraceae). - Bryologist 110: 1-45.

Arnell S. (1956): Hepaticae collected by O. Hedbwrg et al. on the East African Mountains. - Arkiv för Botanik ser. 2, 3(16): 517-562.

Bizot M., Friis I., Lewinsky J. \& Pócs T. (1978): East African bryophytes. IV. Danish collections. Lindbergia 4: 259-284.

Bizot M. \& Pócs T. (1974): East African bryophytes, I. A checklist of bryophytes of East Africa. - Acta Acad. Paedag. Agriensis-Nova 12: 383-449.

- (1979): East African bryophytes III. - Acta Bot. Sci. Hungaricae 25: 223-261.

Bytebier B. \& Chuah-Petiot M.S. (2002): A preliminary checklist of the bryoflora of the Taita Hills, Kenya. - Trop. Bryol. 22: 55-66.

Chuah-Petiot M.S. (1995): Data to the bryoflora of Mount Kenya, Kenya. - Trop. Bryol. 10: 41-54.

- (1996): Bryophytes from the Saiwa Swamp National Park, Kenya. - Trop. Bryol. 12: 63-74.

- (1997): Contributions toward a bryoflora of the Aberdare Range, Kenya. - Trop. Bryol. 13: 57-63.

- (2001): Contribution to the bryoflora of Kenya. - Trop. Bryol. 20: 31-37.

- (2003): Mosses, liverworts and hornworts of Kenya. - Publ. by the Author, Nairobi. 273 pp.

Chuah-Petiot M.S. \& Pócs T. (2003): East African bryophytes XIX. A contribution to the bryoflora of Kenya. - Acta Bot. Hungarica 45: 53-64.

De Sloover J.L. (1986): Note de bryologie africaine XIII. Polytrichaceae. - Bull. Jard. Bot. Nat. Belgique 56: 241-300.

Enroth J., Nyqvist P., Malombe I., Pellikka P. \& Rikkinen J. (2013): Additions to the moss flora of the Taita Hills and Mount Kasigau, Kenya. - Polish Bot. J. 58: 495-510.

Gradstein S.R. (1975): A taxonomic monograph of the genus Acrolejeunea (Hepaticae). - Bryophyt. Biblioth. 4: 1-162, pls. I-XXIV.

- (2012): On the identity of Jungermannia obscura Swartz. - Cryptogamie, Bryologie 33: 257-262.

Grolle R. (1995): The Hepaticae and Anthocerotae of the East African Islands. - Bryophyt. Biblioth. 48: 1-178.

Heinrichs J., Lindner M., Groth H. \& Reinker C. (2005): Distribution and synonymy of Plagiochila punctata (Taylor) Taylor, with hypotheses on the evolutionary history of Plagiochila sect. Arrectae (Plagiochilaceae, Hepaticae). - Pl. Syst. Evol. 250: 105-117.

Herzog T. (1948): Two new African species of Lejeuneaceae. - Trans. British Bryol. Soc. 1: 104-107.

Hyvönen J. (1989): A synopsis of genus Pogonatum (Polytrichaceae. Musci). - Acta Bot. Fenn. 138: 1-87.

Kis G. (1985): Mosses of south-east tropical Africa. An annotated list with distributional data. - Inst. Ecol. Bot. Hungarian Acad. Sci., Vácrátót. 170 pp.

Kruijer H. (2002): Hypopterygiaceae of the world. - Blumea Suppl. 13: 1-388.

Malombe I. (2007): Systematics of Cheilolejeunea (Spruce) Schiffn. (Lejeuneaceae) in continental Africa and its eco-logical signifiance in conservation of Kakamega and Budongo rainforests. PhD-Thesis, Univ. KoblenzLandau. 177 pp.

Jones E.W. (1962): African hepatics XV. Plagiochila in Tropical Africa. - Trans. British Bryol. Soc. 4: 254-325.

Malombe I., Matheka K.W., Pócs T. \& Patiño J. (2016): Edge effect on epiphyllous bryophytes in Taita Hills fragmented afromontane forests. - J. Bryol. 38: 33-46.

Myers N., Mittermeier R.A., Mittermeier C.G., da Fonseca G.A.B. \& Kent J. (2000): Biodiversity hotspots for conservation priorities. - Nature 403: 853-858.

Müller F., Wigginton M.J. \& O'Shea B.J. (2000): New bryophyte taxon records for tropical countries IV. Trop. Bryol. 18: 199-202.

O'Shea B.J. (2006): Checklist of the mosses of sub-Saharan Africa (version 5, 12/06). - Trop. Bryol. Res. Rep. 6: 1-255. Available from: http://www.oshea.demon.co.uk/tbr/afrchk5.pdf

O'Shea B.J., Wigginton M.J., Bruggeman-Nannenga M.A., Hodgetts N.G. \& Porley R.D. (2001): British Bryological Society Expedition to Mulanje Mountain, Malawi. 13. New and other unpublished records. - Trop. Bryol. 20: 1-26.

Pócs T. (2010): On some new or less known Lejeunea (Lejeuneaceae, Jungermanniopsida) species in tropical Africa. East African bryophytes, XXVII. - Nova Hedwigia Beih. 138: 99-116.

Pócs T. \& Luke Q. (2007): East African Bryophytes, XXV. Bryological records from the Chyulu Range, Kenya. - J. East African Nat. Hist. 96: 27-46.

Pócs T. \& Váňa J. (2015): East African Bryophytes XXX. - Acta Biol. Plant. Agriensis 3: 3-21.

Rao P. (2001): Taxonomic studies on Cryphaea (Cryphaeaceae, Bryopsida). 3. Revision of European, African, Australian and Oceanian, and American species. - Bryobrothera 7: 37-111. 
Räsänen M., Chung M., Katurji M., Pellikka P., Rinne J. \& Katul G.G. (2018): Similarity in fog and rainfall intermittency. - Geophys. Res. Lett. 45: 10691-10699.

Shi X.-G, Gradstein S.R \& Zhu R.-L. (2015): Phylogeny and taxonomy of Archilejeunea (Spruce) Steph. (Lejeuneaceae subfam. Ptychanthoideae, Marchantiophyta) based on molecular markers and morphology. Taxon 64: 881-892.

So M.L. (2004): Metzgeria (Metzgeriaceae, Marchantiophyta) in Africa. - New Zealand J. Bot. 42: 271-292.

Tixier P. (1995): Résultats taxonomiques de l'éxpedition BRYOTROP au Zaire et Rwanda. 30. Bryophytes épiphylles (récoltes de E. Fischer). - Trop. Bryol. 11: 11-76.

Touw A. (2001): A review of the Thuidiaceae (Musci) and a realignment of taxa traditionally accommodated in Thuidium sensu amplo (Thuidium Schimp., Thuidiopsis (Broth.) M. Fleisch., and Pelekium Mitt.), including Aequatoriella gen. nov. and Indothuidium gen. nov. - J. Hattori Bot. Lab. 90: 167-209.

Vanden Berghen V. (1965): Hépatiques récoltées par le Dr J.-J. Symoens dans la région péri-tanganyikaise. Bull. Soc. Roy. Bot. Belgique 98: 129-174.

Wigginton M.J. (2018): Checklist and distribution of the liverworts and hornworts of sub-Saharan Africa, including the East African islands. $4^{\text {th }}$ ed. - Trop. Bryol. Res. Rep. 9: 1-138. Downloadable from: http://rbgweb2.rbge.org.uk/bbs/Activities/tbg/resources_lit_africa.htm

Wigginton M.J. \& Grolle R. (1996): Catalogue of the Hepaticae and Anthocerotae of sub-Saharan Africa. Bryophyt. Biblioth. 50: 1-267.

\section{ANNOTATIONS}

Specimen citations and notes for new additions to the flora. All specimens are deposited in $\mathrm{H}$ with many duplicates in Nairobi. The abbreviation JR indicates the collector is Jouko Rikkinen. Coordinates and other data for new datapoints (DP) recorded by Åsa Stam in 2016 are listed here. Vuria: Stam DP 2, 324'53.8"S $38^{\circ} 17^{\prime} 27.1^{\prime \prime E}, 2180 \mathrm{~m}, 21$. Jan. 2016. Stam DP 16, 324'29.9"S 38॰18'33.5"E, $1715 \mathrm{~m}$, 23. Jan. 2016. Stam DP 40, 324'14.8"S 38 17'59.6"E, 1937 m, 24. Jan. 2016. Stam DP 42, 3²4'04.1"S 38¹8'06.1"E, 1836 m, 24. Jan. 2016. Stam DP 48, 322'07.0"S 38 20'28.2"E, 1887 m, 25. Jan. 2016. Stam DP 61, moss-rich tree trunks, 324'59.7"S $38^{\circ} 17^{\prime} 24.1^{\prime \prime E}, 2038 \mathrm{~m}, 25$. Jan. 2016. Stam DP 164, moss-rich moist, indigenous forest, $3^{\circ} 24^{\prime} 52.2^{\prime \prime S} 38^{\circ} 17^{\prime} 33.5^{\prime \prime E}$, 2136 m, 31. Jan. 2016. Yale: Stam DP 70, 3²3'52.6"S 38²0'14.6"E, 1830 m, 26. Jan. 2016. Stam DP 71, 323'53.3"S 38²0'11.5"E, 1808 m, 26. Jan. 2016. Stam DP 73, 3²4'05.5"S 38²0'04.8"E, 1819 m, 26. Jan. 2016. Stam DP 77, 3²4'28.1"S 38¹9'51.8"E, 1827 m, 26. Jan. 2016. Stam DP 78, 324'35.9"S 38¹9'49.5"E, 1796 m,

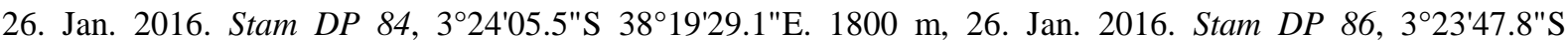
38 19'36.6"E, 1757 m, 26. Jan. 2016. Stam DP 97, 3²4'05.5"S 38¹9'42.9"E, 2075 m, 26. Jan. 2016. Stam DP $175,3^{\circ} 23^{\prime} 56.4^{\prime \prime S} 38^{\circ} 19^{\prime} 54.6^{\prime \prime E}, 1960$ m, 1. Feb. 2016. Ngangao: Stam DP 104, 3²2'07.0"S 38²0'28.2"E, 1843 m, 29. Jan. 2016. Stam DP 107, 3²1'56.4"S 38²0'27.3"E, 1879 m, 29. Jan. 2016. Stam DP 108, 321'53.4"S $38^{\circ} 20^{\prime} 26.6^{\prime \prime E}, 1923 \mathrm{~m}, 29$. Jan. 2016. Stam DP 128, poorly with mosses on tree trunks, 322'05.6"S 38 $20^{\prime} 37.9^{\prime \prime} \mathrm{E}$, 1801 m, 29. Jan. 2016. Stam DP 142, 3²1'43.9"S 38²0'29.0"E, 1863 m, 30. Jan. 2016.

\section{MARCHANTIOPHYTA}

1 Acanthocoleus chrysophyllus. Vuria. Stam VUS C3. - Widely distributed in sub-Saharan Africa, previously reported in Kenya from the Aberdare Mountains and Cherangani Hills (Bizot et al. 1978, as Dicranolejeunea chrysophylla (Lehm.) Grolle).

2 Acrolejeunea emergens. Ngangao. Stam NGPE A3. - Very widely distributed in sub-Saharan Africa, previously reported in Kenya from Mombasa (Herzog 1948, as Ptychocoleus rupestris Herzog; Vanden Berghen 1965, as P. emergens (Mitt.) Steph.) and Malindi and Nairobi (Gradstein 1975).

3 Archilejeunea elobulata. Mbololo. Plot I82. Full shade, moist, fallen from tree. Indigenous forest. Common tree: Newtonia buchananii. $3^{\circ} 20.087^{\prime}$ S, $38^{\circ} 26.977^{\prime}$ E, 1534 m, 26. Jan. 2011, Nyqvist 111370. - Previously known from Angola, Central African Republic, Ivory Coast, South Africa, Tanzania and Zaire. Cited as Dibrachiella elobulata (Steph.) X.Q. Shi, R.L. Zhu \& Gradst. by Shi et al. (2015) and Wigginton (2018).

4 Bazzania nitida. Mbololo. Plot I73. Shade, moist. Collected from rotten log. Indigenous forest. Common tree: Craibia zimmermannii. $3^{\circ} 19.304 '$ S, $38^{\circ} 27.227^{\prime}$ E, 1699 m, 28. Jan. 2011, Nyqvist 10427. - Previously known from 16 countries in the sub-Saharan Africa, nearest to Kenya from Uganda and Tanzania.

5 Calypogeia fissa. Fururu. Plot E58. Partial shade, dry/mesic, sand. Collected from trail side. Exotic forest. Common trees: Eucalyptus sp. $3^{\circ} 25.445^{\prime} \mathrm{S}, 38^{\circ} 19.805^{\prime} \mathrm{E}, 1673 \mathrm{~m}, 24$. Jan. 2011, Nyqvist 111301. Mwachora. JR71817, 9. Sept.9. 2013. - Vuria. JR53322, 6. Dec. 2012; JR74433, 30. Dec. 2013). - Previously known in Kenya from the Aberdare Range (Chuah-Petiot 1997).

6 Cheilolejeunea decursiva. Vuria. Stam VUN A4, VUN B3, VUN C3, VUS B2, VUS C3, VMS C3. - Widely 
distributed from West to East sub-Saharan, previously reported in Kenya from Kakamega forest (Malombe 2007).

7 Cheilolejeunea montagnei. Mbololo. Close to plot I48. Shade, moist. Fallen from trees. Indigenous forest. $3^{\circ}$ 19.576' S, 38²6.928' E, 1681 m, 26. Jan. 2011, Nyqvist 111389. - Previously reported from Kenya by Bizot \& Pócs (1974).

8 Cheilolejeunea serpentina. Mbololo. Plot E40. Partial shade, mesic/moist. Collected from tree base. Exotic forest. Common trees: Eucalyptus sp. $3^{\circ} 20.166^{\prime} \mathrm{S}, 38^{\circ} 26.806^{\prime} \mathrm{E}, 1531 \mathrm{~m}, 26$. Jan. 2011, Nyqvist 111361. Fururu. Plot I37. Partial shade, moist. Collected from rotten log. Indigenous forest. $3^{\circ} 25.801^{\prime} 38^{\circ} \mathrm{S}, 20.419^{\prime} \mathrm{E}$, 1615 m, 20. Jan. 2011, Nyqvist 111792. - Vuria. Stam VMS B3, VMS C3. - Previously reported in Kenya from Kwale in the Shimba Plateau SW of Mombasa (Müller et al. 2000).

9 Cololejeunea grossepapillosa. Vuria. Stam VUN A4, VUN B3, VUS C3. - A Central and East African species previously known from nine countries, nearest to Kenya from Tanzania, Rwanda, Burundi, Uganda, and Ethiopia.

10 Diplasiolejeunea cavifolia. Ngangao. Stam NGNE A3. - Widely distributed in central and East Africa, previously reported from Kenya by Bizot \& Pócs (1974).

11 Diplasiolejeunea kraussiana. Ngangao. Stam NGPW C2, NGPW C3. Wundanyi. Stam RSE C2. - Distributed in East and South Africa, known from 10 countries, nearest to Kenya from Uganda and Tanzania.

12 Drepanolejeunea vesiculosa. Mbololo. Plot E33. Partial shade, moist. Collected from ground. Exotic forest. $3^{\circ}$ 18.911' S, 38²7.399' E, 1713 m, 27. Jan. 2011, Nyqvist 10413. - Tixier (1995) treated D. physifolia (Gottsche) Steph. as a taxonomic synonym of $D$. vesiculosa, but according to Grolle (1995) that synonymy remained "an open question". Drepanolejeunea physifolia has been reported from numerous African countries, also from Kenya (e.g. Wigginton \& Grolle 1996, Wigginton 2018).

13 Frullania obscura. Chawia. Nearby link mast. Open/ partial shade, mesic. Tree trunk/base. Exotic forest. Common trees: Eucalyptus sp. $3^{\circ} 28.4^{\prime} \mathrm{S}, 38^{\circ} 20.4^{\prime} \mathrm{E}, 1572 \mathrm{~m}, 22$. Jan. 2011, Nyqvist 111764. - Ndiwenyi. Plot: I42. Partial shade, moist/mesic. Fallen. Indigenous forest. $3^{\circ} 26.247^{\prime} \mathrm{S}, 38^{\circ} 20.543^{\prime} \mathrm{E}, \mathrm{s} 1595 \mathrm{~m}, 24 \mathrm{Jan}$. 2011, Nyqvist 111332.

14 Frullania obscurifolia. Ngangao. Stam NGEW A3, NGNE A3. Vuria. Stam VUS B2, VUS C3, VLN B3, VMN $C 2$, VMN C3, VUOX C2, VUOX C3, VUOY C3). - Wundanyi. Stam RSW B2, RSW C3, RSE C2). - Very widely distributed in sub-Saharan Africa, known from 30 countries. In Kenya reported from Saiwa Swamp National Park (Chuah-Petiot 1996), Aberdare Mountains (Chuah-Petiot 1997, Chuah-Petiot \& Pócs 2003), Cherangani Hills, Matthews Range and Maparasha Hill (Chuah-Petiot 2001), and Chyulu Range (Pócs \& Luke 2007).

15 Frullania spongiosa. Plot I58. Open, mesic, sand. Collected from tree base. Indigenous forest. $3^{\circ} 21.778^{\prime} \mathrm{S}$, $38^{\circ} 20.545^{\prime} \mathrm{E}, 1787 \mathrm{~m}, 1$ Feb. 2011, Nyqvist 10478. - Previously reported from the Chyulu range in Kenya (Pócs \& Luke 2007).

16 Frullania trinervis. Ngangao. Plot I15. Partial shade, moist. Fallen. Indigenous forest. Common tree: Macaranga capensis. $3^{\circ} 21.394^{\prime}$ S, 38 ${ }^{\circ} 20.349^{\prime}$ E, 1892 m, 1. Feb. 2011, Nyqvist 10481. - Very widely distributed in sub-Saharan Africa, previously reported in Kenya from the Aberdare Mountains (Chuah-Petiot \& Pócs 2003).

17 Herbertus dicranus. Kasigau. JR19004, 3. Feb. 2015. - Vuria. Stam VMN B2; JR51024, 14. Sept. 2012; $J R 38647,27$. Oct. 2016. - Very widely distributed in sub-Saharan Africa, previously known in Kenya from Mt. Elgon (Arnell 1956) and Mt. Kenya (Chuah-Petiot 1995).

18 Lejeunea amaniensis. Mbololo. Plot E40. Partial shade, mesic/moist. Collected from tree base. Exotic forest. Common trees: Eucalyptus sp. $3^{\circ} 20.166 '$ S, $38^{\circ} 26.806 '$ E, 1531 m, 26. Jan. 2011, Nyqvist 111366. - Previously reported from Cameroon, Ethiopia, Madagascar, Rwanda and Tanzania.

19 Lejeunea cyathearum. Fururu. Plot I37. Partial shade, moist. Collected from rotten log. Indigenous forest. $3^{\circ}$ 25.801' S, 38 20.419' E, 1615 m, 20. Jan. 2011, Nyqvist 111792 (with Cheilolejeunea serpentina). - Ngangao. Near car park. Partial shade, moist, sand. Collected from tree base. Indigenous forest. $3^{\circ} 21.270^{\prime} \mathrm{S}, 38^{\circ} 20^{\prime} 16$ E, 1824 m, 18. Jan. 2011, Nyqvist 111739 (with Metzgeria furcata). - Previously known from the Comoro Islands, Democratic Republic of the Congo, Malawi, Rwanda, Tanzania, and Uganda.

20 Lejeunea villaumei. Fururu. Less than $300 \mathrm{~m}$ from plot I37. Partial shade/open, moist. Collected from palm trunk. Exotic forest. $3^{\circ} 25.801^{\prime}$ S, 38 $20.419^{\prime}$ E, 1615 m, 20. Jan. 2011, Nyqvist 111796. Ngangao. Stam NGNE A3. Vuria. Stam VMS B2, VMS B3, VMS C3. - Previously reported from Kenya on Mt. Kenya (Bizot \& Pócs 1979).

21 Lopholejeunea laciniata. Fururu. Plot E59. Partial shade, moist. Collected from trunk/branch. Exotic forest. Common trees: Eucalyptus sp. $3^{\circ} 25.524^{\prime}$ S, 38¹9.941' E, 1724 m, 24. Jan. 2011, Nyqvist 111322. - Previously reported from Malawi, Tanzania and Zimbabwe.

22 Metzgeria crassipilis. Mbololo. Plot I82. Shade, moist. Collected from tree trunk. Indigenous forest. Common tree: Newtonia buchananii. $3^{\circ} 20.087^{\prime}$ S, 38²6.977' E, 1534 m, 26. Jan. 2011, Nyqvist 111368. - Previously known from Burundi, Democratic Republic of the Congo, Malawi, Tanzania, and Uganda.

23 Metzgeria nudifrons. Chawia. Plot E18. Shade, moist. Collected from tree trunk. Exotic forest, 
Tabernaemontana stapfiana. $3^{\circ} 28.449^{\prime}$ S, 38²0.456' E, 1572 m, 22. Jan. 2011, Nyqvist 111757. - Mbololo. Plot I82. Partial, moist. Fallen from trees. Indigenous forest. Common tree: Newtonia buchananii. $3^{\circ} 20.087^{\prime}$ S, $38^{\circ} 26.977^{\prime}$ E, 1534 m, 26. Jan. 2011, Nyqvist 111369. - Previously known from 11 African countries, nearest to Kenya from Uganda and Tanzania.

24 Myriocoleopsis minutissima. Ngangao. Stam NGEW A3. - Vuria. Stam VUOX C2. - Wundanyi (Taita Hills Research Station). Stam RSE B2. - Widely distributed in sub-Saharan Africa, previously reported in Kenya from the Aberdare Mountains (Chuah-Petiot \& Pócs 2003, as Cololejeunea minutissima (Sm.) Steph.).

25 Plagiochila barteri. Mbololo. "Teacher's house", garden, open/partial shade, moist, tree trunk. $3^{\circ} 20.100^{\prime} \mathrm{S}$, 38²6.800' E, 1540 m, 26. Jan.2011, Nyqvist 111360. Plot I48 (<100m), indigenous forest. Common tree: Macaranga capensis. Shade, moist, fallen. $3^{\circ} 19.576^{\prime}$ S, 38²6.928' E, 1681 m, 26 Jan. 2011, Nyqvist 111389. - Vuria. Trail near Vuria's peak. Indigenous forest, open, mesic, sand, tree base. $3^{\circ} 25.278^{\prime} \mathrm{S}, 38^{\circ} 17.345^{\prime} \mathrm{E}$, 1957 m, 19. Jan. 2011, Nyqvist 111819. Near Vuria's peak. Indigenous forest with Coffea sp., Prunus africana. Partial shade, moist/mesic, tree trunk. 3²5.278' S, 38 17.345'E, 2169 m, 19. Jan. 2011, Nyqvist 111822. Stam VUN B3, VUN C3, VUS B2, VUS C3, - In Kenya previously reported from the Aberdare Range (Chuah-Petiot 1997).

26 Plagiochila boryana. Mbololo. Plot I76. Partial shade, moist, sand. Collected from log. Indigenous forest. $3^{\circ} 19.596^{\prime}$ S, 38²7.111' E, 1546 m, 28. Jan. 2011, Nyqvist 10420. - Previously reported from Mauritius, Madagascar, Réunion, Tanzania and Uganda.

27 Plagiochila exigua. Vuria. Stam VUN A4, VUN B3, VUN C3, VUS B2, VUS C2. - Widely distributed in East and southern Africa, in Kenya previously known from the Aberdare Mountains (Chuah-Petiot \& Pócs 2003).

28 Plagiochila kiaeri. Mbololo. Plot E31. Partial shade, moist. Collected from tree base. Exotic forest. Common tree: Macaranga capensis. $3^{\circ} 18.517^{\prime}$ S, 38 27.434' E, 1684 m, 27. Jan. 2011, Nyqvist 10401. - Previously reported from Kenya by Bizot \& Pócs (1974) and Pócs \& Luke (2007, Chyulu Range).

29 Plagiochila moenkemeyeri. Chawia. Plot I25. Partial shade, moist. Collected from tree base. Indigenous forest. $3^{\circ} 28.992^{\prime}$ S, 38²0.652' E, 1545 m, 25. Jan. 2011, Nyqvist 111349. Plot E18. Partial shade, moist. Collected from tree trunk/base. Exotic forest. Common tree: Tabernaemontana stapfiana. $3^{\circ} 28.449^{\prime} \mathrm{S}, 38^{\circ} 20.456^{\prime} \mathrm{E}$, 1572 m, 22. Jan. 2011, Nyqvist 111756. - Mbololo. Plot E83. Partial shade/open, moist. Collected from tree trunk. Exotic forest. Common trees: Pinus sp. $3^{\circ} 23.741^{\prime} \mathrm{S}, 38^{\circ} 19.771^{\prime} \mathrm{E}, 1898 \mathrm{~m}$, 28. Jan. 2011, Nyqvist 10434. Plot 170. Shade, moist. Collected from tree trunk. Indigenous forest. Common tree: Macaranga capensis. $3^{\circ} 20.204^{\prime} \mathrm{S}, 38^{\circ} 26.946^{\prime} \mathrm{E}, 1459$ m, 26. Jan. 2011, Nyqvist 111367. - Vuria. Near Vuria's peak. Open, moist/mesic ("elfin"). Tree trunk. Indigenous forest. $3^{\circ} 25.278^{\prime}$ S, 38 $17.345^{\prime}$ E, 2100 m, 19. Jan. 2011, Nyqvist 111825. - Previously known from 12 African countries, closest to Kenya from Tanzania and Ethiopia.

30 Plagiochila punctata. Mbololo. Plot E33. Partial shade, moist. Collected from ground. Exotic forest. $3^{\circ} 18.911^{\prime} S, 38^{\circ} 27.399^{\prime}$ E. 1713 m, 27. Jan. 2011, Nyqvist 10413. - According to Heinrichs et al. (2005) this widespread species is encountered in East Africa (Democratic Republic of the Congo, Uganda, Tanzania). They did not cite any specimens from Kenya, but is has been reported from the country (Wigginton 2018).

31 Plagiochila terebrans. Fururu. Plot I37 (<300m). Partial shade/open, moist. Collected from palm trunk. Exotic forest. $3^{\circ} 25.801^{\prime}$ S, 38 $20.419^{\prime}$ E, 1615 m, 20. Jan. 2011, Nyqvist 111796. - Ngangao. Plot I15. Partial shade, moist. Collected from tree base. Indigenous forest. Common trees: Macaranga capensis. $3^{\circ} 21.394 ' \mathrm{~S}$, $38^{\circ} 20.349^{\prime}$ E, 1892 m, 1. Feb. 2011, Nyqvist 10482. Stam NGNE A3. - Vuria. Near Vuria's peak. Partial shade, moist/mesic. Tree trunk. Indigenous forest. Common tree: Prunus africana. $3^{\circ} 25.278^{\prime} \mathrm{S}, 38^{\circ} 17.345^{\prime} \mathrm{E}, 2100$ m, 19. Jan. 2011, Nyqvist 111824. Stam VMS B2, VUS B2. - Reported before from Kenya by e.g. Jones (1962).

32 Plicanthus hirtellus. Vuria. Trail near Vuria's Peak. Open, mesic, sand. Collected from tree base. Indigenous forest. $3^{\circ} 25.278^{\prime}$ S, $38^{\circ} 17.345^{\prime} \mathrm{E}, 1957 \mathrm{~m}, 19$. Jan. 2011, Nyqvist 111819. - Recorded in Kenya before from the Aberdare Range (Chuah-Petiot 1997, as Chandonanthus hirtellus).

\section{BRYOPHYTA}

33 Brachymenium nepalense. Maktau Hill (highest peak). $3^{\circ} 22^{\prime} 0.75^{\prime \prime S}, 38^{\circ} 8^{\prime 2} 23.29^{\prime \prime E}, 25$ Oct. 2014, Rikkinen 14650. - Chawia (JR52584, 4. Dec. 2012). - Mwachora (JR71833, 9. Sept. 2013; JR71908, 9. Sept. 2013). Vuria (JR110101, 26. Aug. 2011; JR38960, 31. Oct. 2016). - Known from 12 African countries, closest to Kenya from Rwanda and Zaire.

34 Breutelia stuhlmannii. Kasigau. Along forest path on upper part of N slope, between sample plots 10 and 15, 22 Oct. 2014, JR13902. - Known in Kenya from Mt. Elgon, Mt. Kenya and the Aberdare Range (Kis 1985, Chuah-Petiot 1995, 1997).

35 Chenia leptophylla. Kasigau. Along forest path on lower part of W slope, between sample plots 45 and 50 , 23 Oct. 2014, JR14194. - Previously reported in Kenya from the Chirimo Campus of the Nairobi University (Chuah-Petiot \& Pócs 2003). Treated as Leptophascum leptophyllum (Müll. Hal.) J. Guerra \& M.J. Cano in O'Shea (2006).

36 Cryphaea rutenbergii. Maktau Hill (highest peak). 3²2'0.75"S, 38 8'23.29"E, 25 Oct. 2014, JR14651, JR14659. - Vuria. Stam DP23. - Yale. Stam DP71, DP73. - Previously reported from several localities in 
Kenya (Kis 1985 as C. robusta, Chuah-Petiot 1996, 1997, 2001, Rao 2001)

37 Fissidens androgynus. Kasigau. Along forest path on lower part of W slope, between sample plots 45 and 50, 23 Oct. 2014, JR14179. - Known in Kenya from a few localities (Kis 1985, Chuah-Petiot 2001), previously treated as F. bryoides var. bryoides (O'Shea et al. 2001).

38 Fissidens ovatus. Vuria. JR71811. - This species was previously reported from several localities in Kenya (Chuah-Petiot 2001). It is widely distributed in the sub-Saharan Africa.

39 Funaria hygrometrica. Fururu. JR15403. - Previously (Kis 1985, Chuah-Petiot 1995) reported in Kenya from Limuru near Nairobi, Mt. Kenya and Nyambeni ("Giombene").

40 Hyophila involuta. Kasigau. Along forest path on lower part of W slope, between sample plots 45 and 50 , 23 Oct. 2014, JR14197. - Known in Kenya from Mt. Meru (Kis 1985) and Matthews Range in N Kenya (Chuah-Petiot 2001).

41 Leucophanes hildebrandtii. Kasigau. Plot 56. Moss-rich moist forest with Syzygium guineense, Newtonia buchananii, Ochna holstii, Psychotria petitii and Tabernaemontana stapfiana. UTM 461908, 9577127, 1500 m, 17 Jan. 2010, JR10K566. - Previously reported in Africa from the Comoro Islands, Mauritius, Madagascar, Reunion and Tanzania.

42 Neckeromnion lepineanum. Kasigau. Along forest path on upper part of W slope, between sample plots 50 and 51, 23 Oct. 2014, JR14258. - Reported from 11 African countries, nearest to Kenya from Uganda and Tanzania.

43 Orthostichella pandurifolia was reported by Bytebier \& Chuah-Petiot (2002) from many places in the Taita Hills, but most of the collections may represent $O$. capillicaulis or $O$. rigida. Those two are common in the area, while $O$. pandurifolia is much scarcer (cf. Allen \& Magill 2007).

44 Orthostichopsis subimbricata. Kasigau (JR10K119, JR10K274, JR10K287, JR10K505). - Vuria (JR15022). - Previously known from Cherangani Hills and Kakamega National Park in W Kenya (Bizot et al. 1978). It is distributed in East and Central Africa, as well as in the Comoro Islands, Madagascar and Reunion.

45 Philonotis hastata. Wundanyi. Below Taita Research Station, river bank near concrete dam, JR15016. Very widely distributed in the sub-Saharan Africa, previously known also from several localities in central Kenya (Kis 1985).

46 Pogonatum urnigerum. Hyvönen (1989) did not cite this species for Kenya. It is known from Cameroun, Democratic Republic of the Congo, Rwanda, Tanzania, and Uganda, and has been reported from several localities in Kenya (Kis 1985, De Sloover 1986, Chuah-Petiot 1995, Bytebier \& Chuah-Petiot 2002).

47 Rhodobryum ontariense. Kasigau. Along forest path on upper part of N slope, between sample plots 10 and 15 , 22 Oct. 2014, JR13829. - Ngangao (JR090128, 22. Jan. 2009; JR090104, 20. Jan. 2009; JR50667, 13. Sept. 2012; JR50778, 13. Sept. 9.2012; JR15188, 29. Oct. 2014). - Vuria (JR110113, 26. Aug. 2011; JR50858, 14. Sept. 2012; JR39364, 9. Dec. 2016). - Yale (JR26410, 4. Oct. 2015; JR26474, 4. Oct. 10.2015; JR26517, 4. Oct. 2015). - Reported from seven African countries, the previously knwon Kenyan locality being in the Aberdare Range (Kis 1985).

48 Rhynchostegiella holstii. Ngangao. Stam DP 128. - Reported from six African countries, in Kenya from Nairobi (Kis 1985).

49 Rhynchostegium buluense. Vuria. Stam DP 61. - Previously known from Gabon and Tanzania.

50 Rigodium toxarion var. toxarion. Vuria. Stam DP 164. - Reported from six mainland African countries and Madagascar, in Kenya from Mount Kenya (Chuah-Petiot 1995).

Authors' addresses: Johannes Enroth, Petri Nyqvist \& Åsa Stam, Organismal and Evolutionary Biology Research Programme, P.O. Box 65, 00014 University of Helsinki, Finland Corresponding author Johannes Enroth, e-mail: johannes.enroth@helsinki.fi (other affiliation: Botany Unit, Finnish Museum of Natural History, University of Helsinki, Finland).

Tamás Pócs, Department of Botany, Institute of Biology, Eszterházy Károly College, Eger, pf. 43, H-3301 Hungary.

Xiaolan He \& Jouko Rikkinen, Botany Unit, Finnish Museum of Natural History, P.O. Box 7, 00014 University of Helsinki, Finland.

Itambo Malo mbe, East African Herbarium, National Museums of Kenya, P.O. Box 40658, 00100 Nairobi, Kenya.

Jouko Rikkinen, Botany Unit, Finnish Museum of Natural History \& Organismal and Evolutionary Biology Research Programme, P.O. Box 7, 00014 University of Helsinki, Finland. 